\title{
Enhanced signal detection algorithm using trained neural network for cognitive radio receiver
}

\author{
Sheetal D. Borde ${ }^{1}$, Kalyani R. Joshi ${ }^{2}$ \\ ${ }^{1}$ Department Electronics and Telecommunications Engineering, College of Engineering, India \\ ${ }^{2}$ Department Electronics and Telecommunications Engineering, PES's Modern College of Engineering, India
}

\begin{tabular}{l} 
Article Info \\
\hline Article history: \\
Received Apr 15, 2018 \\
Revised Aug 1, 2018 \\
Accepted Aug 29, 2018 \\
\hline
\end{tabular}

Keywords:

Artificial neural network

Cognitive radio

Cyclostationarity

Enhanced signal detection

Spectrum sensing

\begin{abstract}
Over the past few years, Cognitive Radio has become an important research area in the field of wireless communications. It can play an important role in dynamic spectrum management and interference identification. There are many spectrum sensing techniques proposed in literature for cognitive radio, but all those techniques detect only presence or absence of the primary user in the designated band and do not give any information about the used modulation scheme. In certain applications, in cognitive radio receiver, it is necessary to identify the modulation type of the signal so that the receiver parameters can be adjusted accordingly. Most of the modulated signals exhibit the property of Cyclostationarity that can be used for the purpose of correct detection of primary user and the modulation type. In this paper, we have proposed an enhanced signal detection algorithm for cognitive radio receiver which makes use of cyclostationarity property of the modulated signal to exactly detect, the modulation type of the received signal using a trained neural network. The algorithm gives better accuracy of signal detection even in low SNR conditions. The use of a trained neural network makes it more flexible and extendible for future applications
\end{abstract}

Copyright (C) 2019 Institute of Advanced Engineering and Science. All rights reserved.

\section{Corresponding Author:}

Sheetal D. Borde,

Department of Electronics and Communication Engineering,

College of Engineering, Pune-5, Pune University,

Pune, Maharashtra- 411005, India.

Email: sdbpune@gmail.com

\section{INTRODUCTION}

The existing wireless communication systems and networks are operating based on fixed spectrum assignment policy laid down by regulatory authorities. The exclusive radio frequency spectrum assignment, which is licensed by government regulatory bodies, is an efficient way for interference mitigation. However, the fixed spectrum assignment policies lead to inefficient use of spectrum since most of the channels actively transmit the information only for short duration while the certain portion of the spectrum remains idle for most of the time when the licensed primary users are not transmitting any data [1]. This indicates that the inefficient radio spectrum usage has been a serious bottleneck for deployment of upcoming wireless systems. From this discussion it can be noted that the scarcity of RF spectrum is not a result of lack of spectrum but a result of static spectrum allocations and thereby inefficient usage of the available spectrum. In order to overcome the spectrum scarcity problem, recently Cognitive Radio (CR) system has been proposed to facilitate the spectrum sharing so that the upcoming wireless radio systems can be accommodated without assigning a new spectrum band. The popularity of Software Defined Radio and Cognitive Radio technologies 
is increasing over past few years. The tremendous growth in the wireless communications has led to a huge demand on the implementation of new wireless services in both licensed and unlicensed frequency spectrum. Recent studies show that the fixed spectrum assignment policy results in poor spectrum utilization [1], [2]. Cognitive Radio (CR) is a promising technology to enable access to unoccupied spectrum bands, called white spaces or spectrum holes, thereby achieving efficient spectrum utilization.

There are many spectrum sensing algorithms proposed for spectrum sensing in cognitive radio. In [2] and [3], various spectrum sensing techniques are discussed. The basic three spectrum sensing methods that are usually used are Match filter detection (MFD), Energy Detection (ED) and Cyclostationary Feature Detection (CFD). But most of these methods mainly help to detect the presence or absence of Primary User (PU) by detecting the energy of the sensed signal or by detecting cyclic feature. They do not give any information about the modulation scheme used. Sometimes for a cognitive radio receiver it is not only sufficient to just detect the presence of the signal but also to detect its modulation type and then demodulate that signal to get back the original information signal. In CR receiver, identification of correct modulation scheme of Primary User (PU) is often a critical issue when there is no prior knowledge about signal power, carrier frequency etc. [4].

This work presents an enhanced spectrum sensing algorithm to addres the above discussed problem. The main purpose of the proposed Enhanced Signal Detection (ESD) algorithm for a cognitive radio receiver is not only to identify the existence of a signal in a given frequency band, at a given location, at a given time but also to correctly identify the modulation scheme used to modulate that signal. As we know, modulation is the process of varying a periodic waveform in order to use that signal to convey a message, the algorithm makes use of this periodic signal to confirm the presensce primary user signal. This is because, noise signal do not contains any kind of periodicity, and it is totally random in nature. So, detection of periodicity or cyclostationary feature almost confirms the presence of a modulated carrier which might be carrying some information signal of the primary user. After detection of periodicity, the algorithms further facilitates for the detection of the modulation scheme of the detected signal. Thus, the proposed ESD algorithm using Trained Nueral Network (TNN) provides an intermediate step between signal detection and demodulation. It plays an important role in dynamic spectrum management and interference identification for civilian, commercial and military applications.

\section{LITERATURE REVIEW}

A large number of automatic modulation classification methods have been developed as per the research of at least two decades. Some modulation classification algorithms are based on the use of signal cyclostationarity that falls under the category of feature based methods. In [1], the author has introduced a spectral correlation theory for cyclostationary time series.Second order periodicity of the signal is exploited using cyclostationary properties. A brief survey of the literature on cyclostationarity and the applications of cyclostationarity in communication, signal processing and many other research areas are described in [4]. The authors in [5] have used the Spectral Correlation Function (SCF) and Cyclic Domain Profile (CDP) for signal detection and hidden Markov model for signal classification to process extracted signal features due to its strong pattern-matching capability. The definitions of SCF and CDP are also mathematically explained in [5]. In [6] and [7], the authors have presented signal classification using cyclic spectral analysis and the simulation results of different modulation types under various noise conditions. In [8], the author has discussed how the cyclostationary features in the signals modulated using different schemes can be detected correctly. In [9] authors have suggested the Likelihood-Based Approach to Modulation Classification. In [10] and [11], the authors have discussed the basics of neural networks, their classification and applications in pattern recognition. The objective of paper [12] and [13] is to summarize the methods used in various stages of a pattern recognition system and advantages of Back Propagation Algorithm over other techniques to recognize the complex patterns along with other real-life applications.

The authors have given the overview of Artificial Neural Network, its working principle and training in [14] and [15]. In [16], the authors have provided comprehensive fundamentals in detailed manner with examples and computer-oriented experiments. Moreover, the concepts associated with neural networks are explained. The mathematical treatment of layered neural networks is given in detail. In [17], the author has introduced supervised learning algorithm named Scaled Conjugate Gradient (SCG) and its performance is compared to that of standard Back-Propagation Algorithm. According to [18], the spectral correlation analysis can be preferably applied for signals that are produced by some periodic modification or modulation of stationary random noise. In [19], the author investigates the spectral features of several basic analog and digital modulated signals from point view of modulation recognition and parameter estimation. In [20] and [21], the spectral correlation function that is a generalization of the power spectral density function is described. Explicit formulae for the spectral correlation function for various types of analog and digital

Int J Elec \& Comp Eng, Vol. 9, No. 1, February 2019 : 323 - 331 
modulated signals are also derived. In [22], authors have provided an experimental performance evaluation of the Energy Detector based sensing using NI USRP-2930, which is a Software Defined Radio (SDR) transceiver. In [23], problem of future wireless network has been examined and new learning scheme using artificial neural network is proposed.

The author of [24] has discussed a modulation classification method capable of classifying MFSK digital signals without a priori information using modified covariance method. In reference [25] cyclostationary based spectrum sensing method and a modified intrusion elimination (AIC) algorithm to mitigate the interference between the primary and cognitive users had been proposed. Thus from this literature review, it is clear that cognitive radio is apromising technology to overcome the problem of spectrum scarcity and spectrum sensing is an very important aspec $t$ of cognitive radio receiver. There are various method of spectrum sensing and very few help in recognising parameters of primary signal which is of great importance in certain applications. The proposed algorithm in this paper helps to overcome the problem of correct detection of the primary user presence as well as modulation type. Thus it is not merely a spectrum sensing algorithm but going beyond that to give additional information about primary user, hence the named as enhanced spectrum sensing algorithm.

\section{CYCLOSTATIONARITY, SCF AND CDP}

A cyclostationary process has statistical properties that vary periodically over time. Cyclostationary feature detection method deals with the inherent cyclostationary properties or features of the signal. Such features have a periodic statistics and spectral correlation that cannot be found in any interference signal or stationary noise. This periodicity in the received primary signal is exploited to identifythe presence of primary users, and that is why the cyclostationary feature detection method possesses higher noise immunity than any other spectrum sensing method. In this method, the cyclic spectral correlation function is the parameter that is used for detecting the primary user signals.

Cyclostationary spectrum sensing method performs better than other detection schemes such as energy detection method in low SNR regions, because of its noise rejection capability. This occurs because noise is totally random and does not exhibit any periodic form of behaviour. When we have no prior knowledge about primary user's waveform, which is the scenario in real life, then best technique to be adopted is cyclostationary feature detection. As an advantage, the cyclostationary spectrum sensing method can be used to find out the type of modulation scheme used by the primary user signal.

Spectral correlation function (SCF) is a powerfultool to study cyclostationary signals. It can be used to analyze and extract information from modulated signals in noise according to their cyclostationarity [19]. The expression for SC can be derived as follows:

Signal $x(t)$ satisfies second order periodicity with cyclic frequency $\alpha \neq 0$ if and only if

$$
\widehat{\mathrm{R}}_{\mathrm{x}}^{\alpha}=\lim _{\mathrm{T} \rightarrow \infty} \frac{1}{\mathrm{~T}} \int_{-T / 2}^{\mathrm{T} / 2} \mathrm{x}\left(\mathrm{t}+\frac{\tau}{2}\right) \mathrm{x}\left(\mathrm{t}-\frac{\tau}{2}\right) \mathrm{e}^{-\mathrm{i} 2 \pi \alpha \mathrm{t}} \mathrm{dt}
$$

exists and is not identically zero as a function of $\tau$. Above equation is known as the cyclic autocorrelation (also called limit cyclic autocorrelation). When $\alpha=0$, it reduces to conventional autocorrelation.

We know that, the Power Spectral Density (PSD) is obtained by taking the Fourier transform of the autocorrelation function. Mathematically,

$$
S_{x}(f)=\int_{-\infty}^{\infty} R_{x}(\tau) e^{-i 2 \pi f \tau} d \tau
$$

Similarly, the Spectral Correlation Density (SCD) is the Fourier transform of the cyclicautocorrelation function and is given by,

$$
S_{x}^{\alpha}(f)=\int_{-\infty}^{\infty} R_{x}^{\alpha}(\tau) e^{-i 2 \pi f \tau} d \tau
$$

When $\alpha=0$, (3) reduces to (2).

For practical implementation, Cyclic Periodogram can be defined as [5],

$$
\mathrm{S}_{\mathrm{XT}}^{\alpha}(\mathrm{f})=\frac{1}{\mathrm{~T}} \mathrm{X}_{\mathrm{T}}\left(\mathrm{t}, \mathrm{f}+\frac{\alpha}{2}\right) \mathrm{X}_{\mathrm{T}}^{*}\left(\mathrm{t}, \mathrm{f}-\frac{\alpha}{2}\right)
$$

Where $\mathrm{X}_{\mathrm{T}}$ is the time-variant Fourier transform defined as follows:

$$
X_{T}(t, f) \triangleq \int_{t-T / 2}^{t+T / 2} x(u) e^{-i 2 \pi f} d u
$$


The estimated SCF obtained by frequency smoothing of theCyclic Periodogram in (4) is,

$$
\mathrm{S}_{\mathrm{xT}}^{\alpha}(\mathrm{t}, \mathrm{f})_{\Delta \mathrm{f}} \triangleq \frac{1}{\Delta \mathrm{f}} \int_{\mathrm{f}-\Delta \mathrm{f} / 2}^{\mathrm{f}+\Delta \mathrm{f} / 2} \mathrm{~S}_{\mathrm{xT}}^{\alpha}(\mathrm{t}, \mathrm{v}) \mathrm{dv}
$$

The SCF can be obtained by increasing observation lengthT and reducing the size of the smoothing window $\Delta \mathrm{f}[5]$,

$$
S_{x}^{\alpha}(f)=\lim _{\Delta f \rightarrow 0} \lim _{T \rightarrow \infty} S_{x T}^{\alpha}(t, f)_{\Delta f}
$$

The SCF is a cross-correlation function between frequency components separated by $(\mathrm{f}+\alpha / 2)$ and $(f-\alpha / 2)$. If $x(t)$ contains no spectral components at $f= \pm \alpha / 2$, then the SCF is actually the covariance of the two spectral components. We can define the correlation coefficient for the SCF, which is known commonly as spectral coherence (SC). Mathematically, the spectral coherence is defined as [2], [5], [6]

$$
C_{X}^{\alpha}(f) \triangleq \frac{S_{X}^{\alpha}(f)}{[S(f+\alpha / 2) S(f-\alpha / 2)]^{1 / 2}}
$$

The magnitude of the SC ranges from 0 to 1 . It allows us to measure thestrength of second-order periodicity contained within a timeseries in a simplified way. When SCF is plotted, the occupancy status of the spectrum can be found out. If a primary user signal is present in the operating frequency range, the SCF gives a peak at its centre. The peak will not be present in the case when there is no primary user signal present in the concerned frequency range [7].

However the use of SC requires large amounts of data and hence one of the solutionsis to use only the peak values in the SC. These peak values in the SC are called as cyclic frequency domain profile or simply cyclic domain profile (CDP) or $\alpha$-profile [5]. The cyclic frequency is inherently a discrete parameter. It is convenient to assess the cycle-frequency parameter by evaluating the maximum over spectral frequency $\mathrm{f}$.

$$
\mathrm{I}(\alpha) \triangleq \max _{\mathrm{f}}\left|\mathrm{C}_{\mathrm{x}}^{\alpha}(\mathrm{f})\right|
$$

Neural networks trained using the cyclic domain profiles (CDP) can be used for signal classification due to their patternmatching capabilities. Training the neural network with different realizationsof the signal allows it to extract features such as carrierand keying-rate features of the signal. When the neuralnetwork is trained with a variety of signal realizations withdifferent SNRs, the network performs exceptionally, evenat low SNR levels.

\title{
4. THE PRPOSED ALGORITHM
}

The proposed Enhanced Signal Detection (ESD) algorithm primarily aims at estimation of modulation scheme of the received PU signal. Most modulated signals exhibit the property of Cyclostationarity that can be exploited for the purpose of classification. A feature-based method called Cyclostationary Feature Detection is able to identify different modulation schemes. The Spectral Correlation Function obtained from the sensed signal is used as a cyclic feature. The Cyclic frequency Domain Profile (CDP) derived from Spectral Correlation Function is used as a discriminator in the classification process since several modulation schemes have unique cyclic frequency domain profiles. The neural network approach based on the learning mechanism is employed for pattern matching. It is used for classification of data patterns and distinguishing them into predefined set of classes. The two layered neural network is trained using the Back Propagation Algorithm.

Enhanced Signal Detection (ESD) Algorithm using a Trained Neural Network (TNN)

\author{
Step 1: Detect the noisy modulated signal. \\ Step 2: Estimate its Cyclic Periodogram. \\ Step 3: Perform Time Smoothing. \\ Step 4: Perform Frequency Smoothing. \\ Step 5: Calculate Spectral Coherence. \\ Step 6: Calculate Cyclic Domain Profile. \\ Step 7: Train the Neural network with various Cyclic Domain Profile patterns. \\ Step 8: Obtain Classification Confusion Matrix. \\ Step 9: Verify the results for different modulation schemes.
}


The main Design Considerations for the Enhanced Signal Detection (ESD) Algorithm using a Trained Neural Network (TNN) are as follows:

a. Channel is assumed to be noisy with Additive White Gaussian Noise (AWGN) with varying values of standard deviation.

b. Number of data symbols are 2000 and symbol rate is $3 \mathrm{GHz}$.

c. Sampling frequency selected satisfied Nyquist criterion.

d. FFT is used for time smoothing.

e. Raised Cosine filter is used for frequency smoothing.

f. $400 \mathrm{CDPs}$ of different modulation schemes are input to the neural network.

g. Number of hidden neurons is 10 .

\section{RESULTS AND DISCUSSIONS}

The LabVIEW software from National Instruments is a powerful simulation tool which makes use of graphical programming language. The proposed algorithm is simulated with combined use of LabVIEW and MATLAB. Different types of synthetic signal realisaions are generated to prepare a database to train the ANN. For each of the case SCF and CDP signals are studied. After analysis of the CDP plot for the different synthetic signal realization, the modulation type of the received signal can be recognized. CDP plot is actually a two dimentinal view of the SCF plot which can represent the important characteristics of the signal of our interest with less data samples.

Training the neural networks with different realizations of the signal allowed them to extract and pick out the carrier and cyclostationary features of the signal from the background noise. Time constraints limited the amount of data we were able to generate to test and train the ANN, however considering its sufficient performance with a considerable observation window (on the order of 256 samples) we believe it is possible for this type of network to classify signals with low SNRs and fewer observed samples while preserving the levels of performance and computational complexity.

As of now, we have tested here some specific modulation types using the proposed algorithm, but other advanced modulation schemes can also be detected in future. Based on the some typical nature of SCF and CDP plots, different modulations schemes are discussed in following cases.

Case 1: Noisy Signal: If the received signal consists of only noise (assumed AWGN for simulation), then no signal peaks are observed in the SCF as well as CDP plots. This indicates the absence of signal in the sensed channel i.e. the primary user is absent. This is because noise signal do not contain any form of periodicity, it is totally random in nature.

Case 2: Binary Phase Shift Keying (BPSK) Signal: When a BPSK modulated signal is sensed, then three distinct peaks along $\alpha$ axis are observed, as shown in Figure 1. The pick signal at a perticular frequency represents the maximum signal content at that frequency. As there are only two frequencies, one is information signal and other is career signal in BPSK, the first peak corresponds to the symbol rate $\mathrm{f}_{\mathrm{r}}$, the second one corresponds to the carrier frequency $f_{c}$ and the last one corresponds to $f_{c}+f_{r}$.
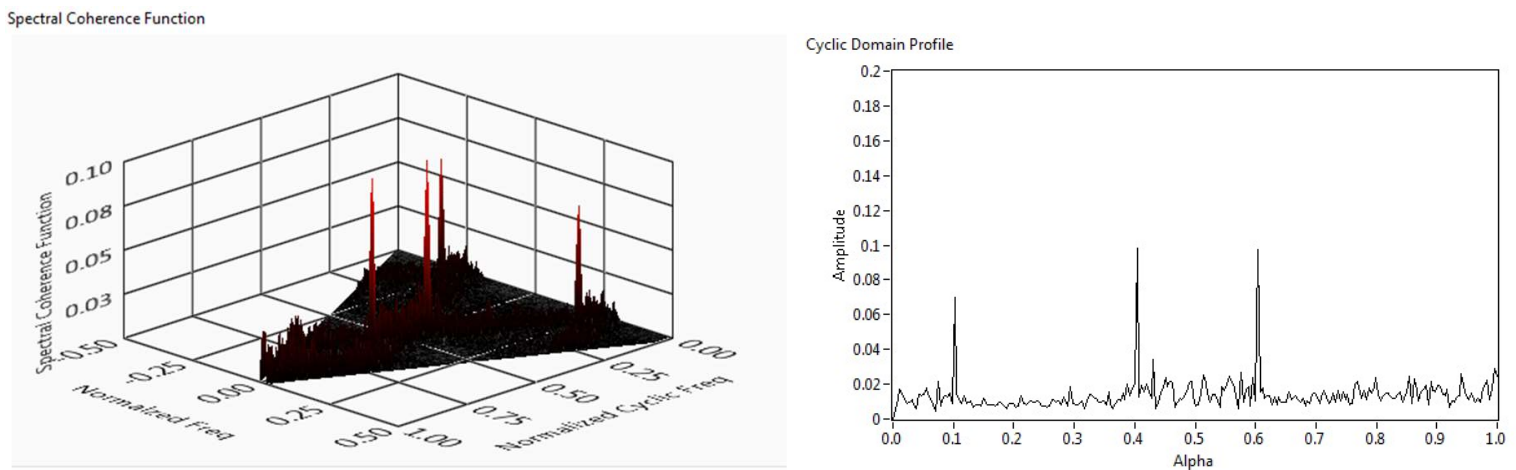

Figure 1. SCF and CDP for BPSK modulated signal showing presence of three peaks

Case 3: Quadrature Phase Shift Keying (QPSK) Signal: When the sensed signal is QPSK modulated, the CDP plot is very simplified with presence of only one peak along $\alpha$ axis, which corresponds to the symbol rate $f_{r}$ as shown in Figure 2. The extra two peaks in BPSK are missing in QPSK, because, 
QPSK is a balanced modulation (has both, in phase and quadrature phase components) and hence peaks corresponding to the carrier frequency get cancelled out.
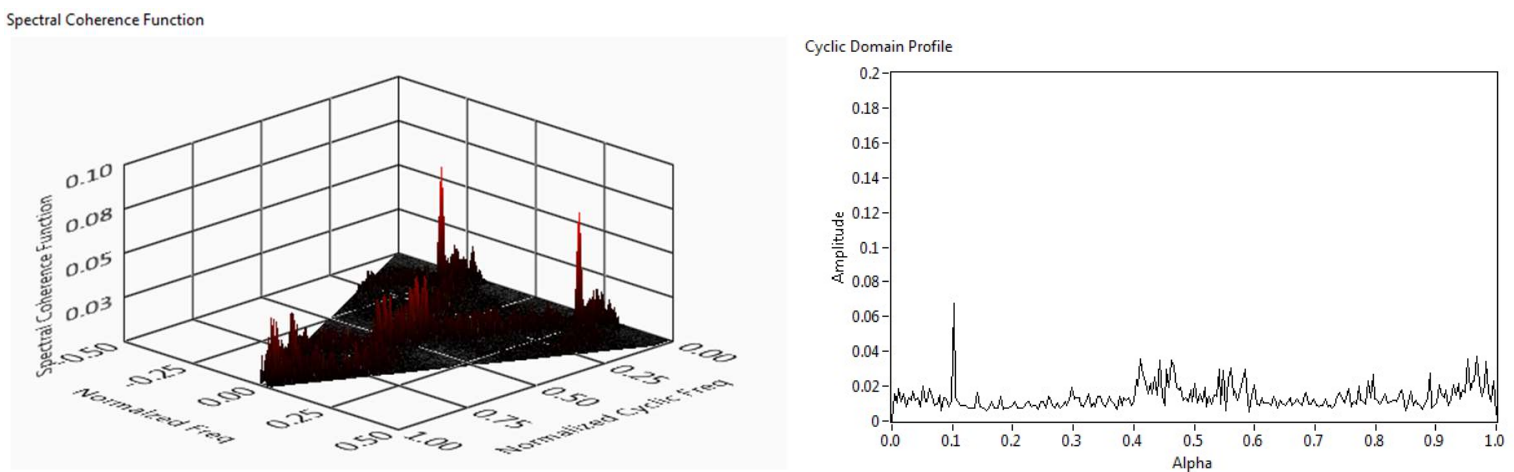

Figure 2. SCF and CDP for QPSK modulated signal showing presence of one peak

Case 4: Frequency Shift Keying (FSK) Signal: If the sensed signal is FSK modulated, there are two impulses of equal amplitude in the CDP as shown in Figure 3, which corresponds to two carrier frequencies involved, i.e. 'mark' frequency and 'space' frequency. Also, the two peaks that overlap the two impulses are observed. These two peaks along with the third one are analogous to the one observed in CDP of BPSK.
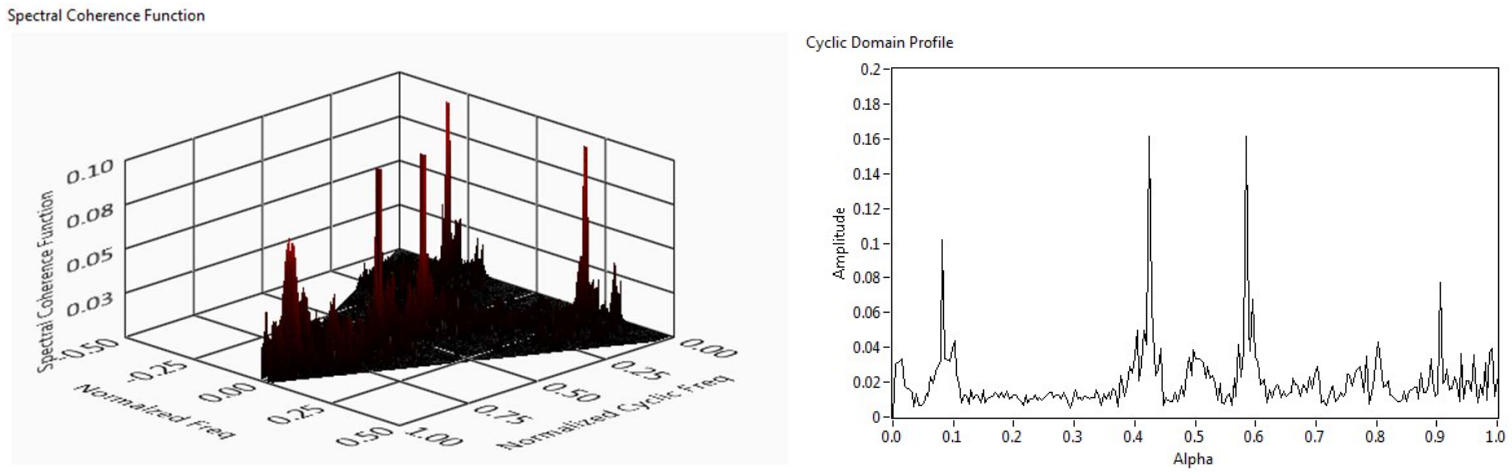

Figure 3. SCF and CDP for FSK modulated signal showing presence of two main peaks

Case 5: Minimum Shift Keying (MSK) Signal: When the received signal is MSK modulated, then the SCF and CDP shows only two peaks as shown in Figure 4. These peaks correspond to the two quadrature carrier $\operatorname{Sin} 2 \pi$ and $\operatorname{Cos} 2 \pi$. This enables easy identification of the MSK signal.
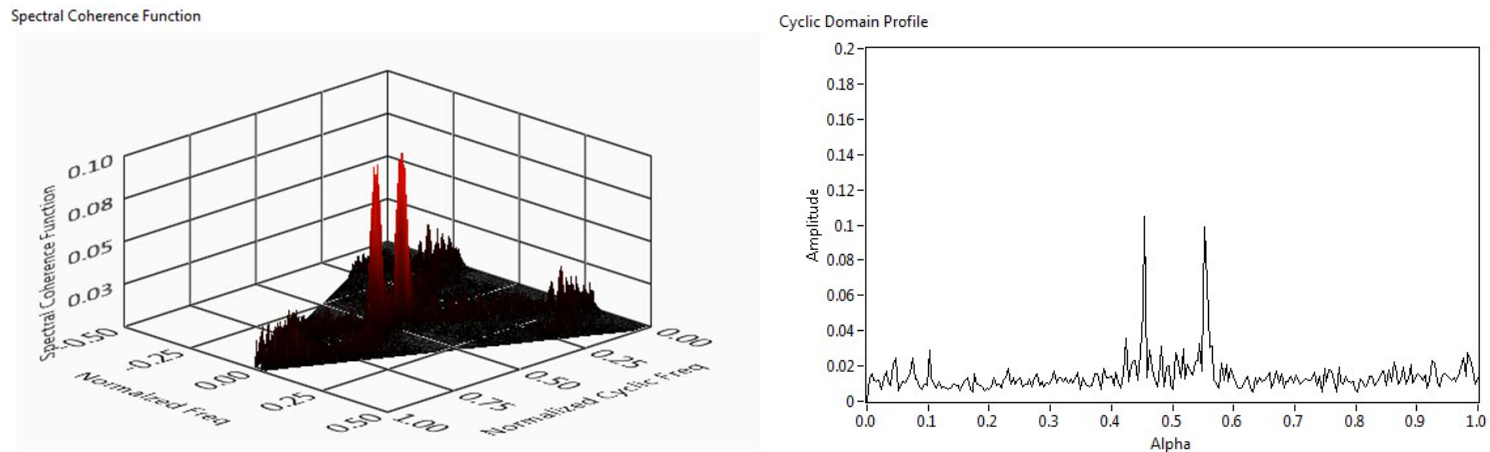

Figure 4. SCF and CDP for MSK modulated signal showing presence of only two peak 
The generated CDPs from the synthetic modulated signals are then given to an artificial neural network. The use of ANN makes it simpler to classify the CDPs and then identify correctly the modulation scheme after training phase. Figure 5 shows the block diagram of the artificial neural network. It is trained with 256 point CDPs of various synthetic modulated signals. There is one hidden layer and one output layer.

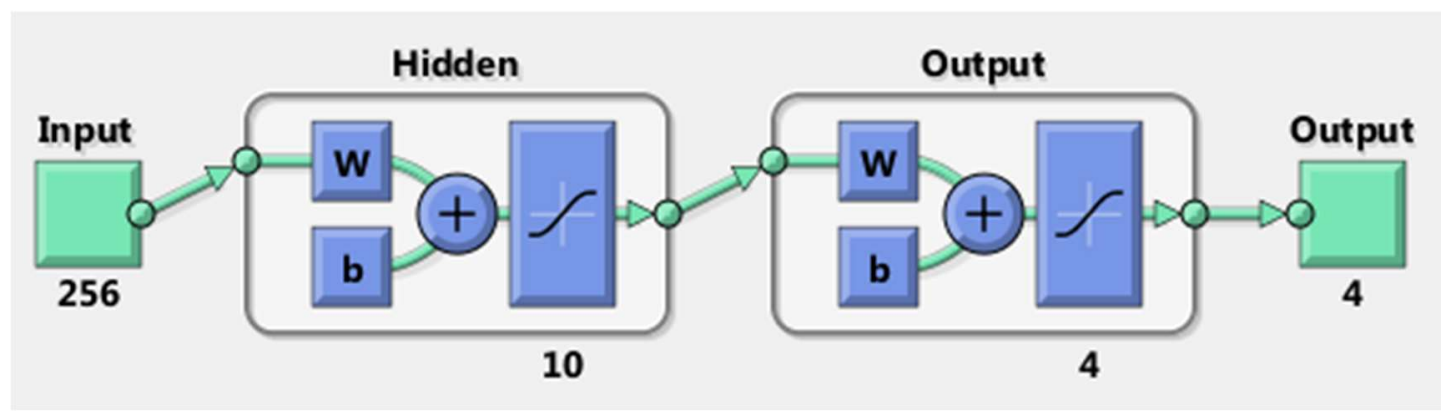

Figure 5. Neural network trained using 256 point CDPs

Figure 6 shows a plot of mean square error versus number of training iterations (epochs) according to which the best validation performance is 0.00026501 at epoch 36 . It can be concluded from the plot that the mean square error decreases with increase in number of epochs and attains a stable value at some instant.

Figure 7 is called Classification Confusion Matrix for training, validation, testing as well as overall classification result. It shows the various types of errors that occurred for the final trained network. In other words, it represents the accuracy of classification. The diagonal cells show the number of cases that were correctly classified (in green) and the off-diagonal cells show the misclassified cases (in red). The blue cell in the bottom right shows the total percentage of correctly classified cases and the total percent of misclassified cases. For training, the accuracy achieved is $98.9 \%$, for training, it is $98.3 \%$ for testing and $100 \%$ for validation. The results show very good recognition since overall accuracy of all confusion matrix is $99 \%$. Thus, the network performs exceptionally well, even at low SNR levels.

The proposed work over here is much different than [4] and [6], as they make use of Hidden Markov Model (HMM) and MAXNET based classifier. It is much different from the work proposed in [23] which focuses mainly on the spectrum sensing of predicted free channels using ANN. Instead, in this work we have proposed an enhanced algorithm for signal detection and classification using ANN which is implemented using LabVIEW and Matlab softwares. This approach firstly helps to fully automise the process of signal classification and secondly, provides flexibility for future enhancements for detection of advanced modulation schemes. The accuracy of classification achieved with this algorithm is almost $100 \%$ which was not achieved with any of the past research work mentioned in the references. These are the some noteworthy contributions of this work and will be proved useful for development of efficient cognitive radio systems in future.

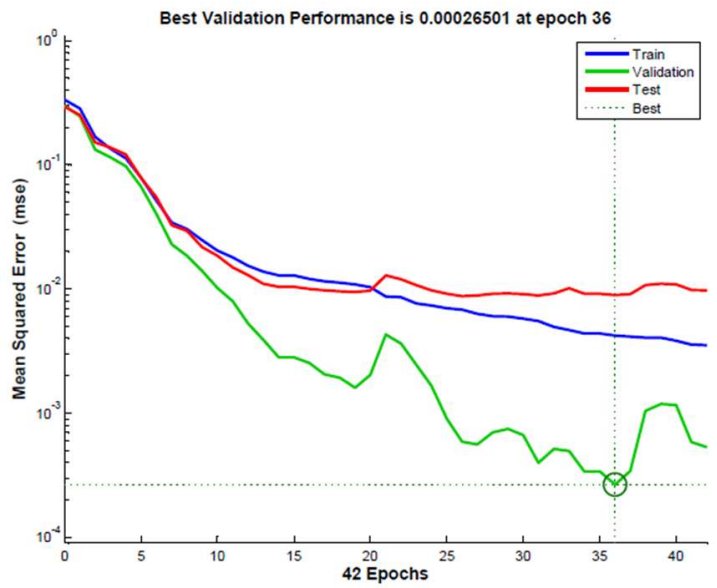

Figure 6. Training, validation and test performance plot 

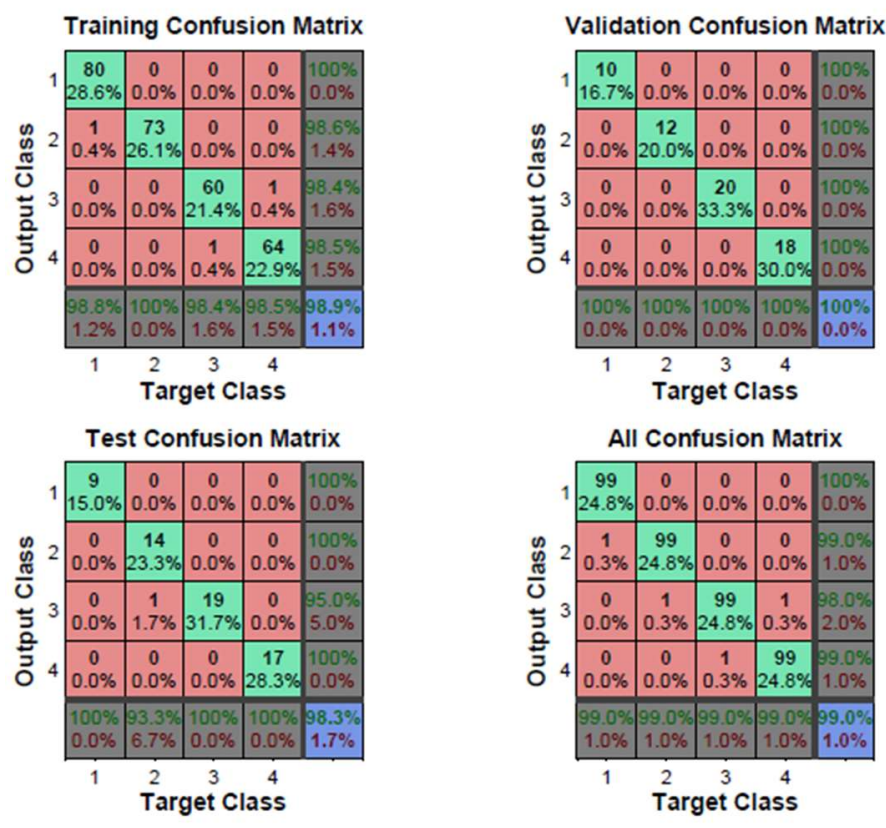

Figure 7. Classification confusion matrix

\section{CUNCLUSION}

The proposed Enhanced Signal Detection algorithm using Trained Neural Network not only helps to detect the signal but classifies it correctly and identifies the modulation scheme. Because of use of cyclostationarity feature of the PU signal, the algorithm can work well even in low SNR conditions and still gives more accurate results as compared to energy detection scheme whose performaaance degrades in noisy environment. The calculated SCF and then CDP of the received signal helps to correctly identify the type of the modulation scheme with the help of neural network. The results show very good recognition rate since overall accuracy of all confusion matrix is $99 \%$. The classification accuracy of the algorithm is also $98.3 \%$ which is the highest among the other classification schemes discussed in literarture review. The use of LabVIEW software for implementation of ANN provides automization of signal classification process and gives the flexibility for future adaptations. It also reduces computational complexity. The proposed algorithm not only gives the assurance of the presence of signal by detecting its scheme of modulation but also helps to demodulate the signal by knowing its type. In this paper, the performance of the ESD algorithm is tested by making use of limited number of modulation schemes, but in future more modulation schemes can be considered. The neural network gives the flexibility for training with advanced modulation schemes such as OFDM, DVB using fading channel environment with noise uncertainty which can further extend the scope of this algorithm in future.

\section{REFERENCES}

[1] W. A. Gardner, "The Spectral Correlation Theory of Cyclostationary Time-Series", Signal Processing, vol.11, no.1, pp. 13-36, 1986.

[2] T. Yucek and H. Arslan, "A Survey of Spectrum Sensing Algorithms for Cognitive Radio Applications," IEEE Comm. Survey Tuts., vol. 11, no. 1, pp. 116-130, 2009.

[3] S. D. Borde, K. R. Joshi, "Signal Processing Techniques for Spectrum Opportunities and Interference Detection in Cognitive Radio Networks", proceedings of IET International Communication Conference of Wireless Mobile \& Computing, Nov 2011.

[4] B. Ramkumar, "Automatic Modulation Classification for Cognitive Radios using Cyclic Feature Detection”, IEEE Circuits and Systems Magazine, vol. 9, no. 2, pp. 27-45, 2009.

[5] W. A. Gardner et al., "Cyclostationarity: Half a century of research", Signal Processing, vol. 86, pp. 639-697, 2006.

[6] K. Kim, I. A. Akbar, K. K. Bae, J.-S. Um, C. M. Spooner, and J. H. Reed, "Cyclostationary Approaches to Signal Detection and Classification in Cognitive Radio," in Proc. IEEE DySpan, pp. 212-215, 2007.

[7] A. Fehske, J. Geaddert, and J. H. Reed, "A New Approach to Signal Classification Using Spectral Correlation and Neural Networks," in Proc. IEEE Int. Symp. New Frontiers in DySpan, 2005. 
[8] Aparna P.S., M. Jayasheela, "Cyclostationary Feature Detection in Cognitive Radio Using Different Modulation Schemes”, International Journal of Computer Applications, vol. 47, no.21, pp. 12-16, 2012.

[9] F. Hameed, O. A. Dobre, D. C. Popescu, "On the Likelihood-Based Approach to Modulation Classification”, IEEE transactions on wireless communications, vol. 8, no. 12, pp. 5884-5892, 2009.

[10] Andrej Krenker et al., "Introduction to the Artificial Neural Networks", Artificial Neural Networks Methodological Advances and Biomedical Applications, 2011.

[11] B. Yegnanarayana, "Artificial Neural Networks for Pattern Recognition”, Sadhana, Vol19, Part 2, April 1994

[12] Dr. Rama Kishore and TaranjitKaur, "Backpropagation Algorithm: An Artificial Neural Network Approach for Pattern Recognition" International Journal of Scientific \& Engineering Research, vol. 3, no. 6, June 2012.

[13] TaranjitKaur, "Implementation of Backpropagation Algorithm - A Neural Network Approach for Pattern Recognition" International Journal of Engineering Research and Development, vol. 1, no. 5, pp. 30-37 https://pdfs.semanticscholar.org/b0a6/578f40c4f58174670c755a7a084fa8a0c471.pdf, June 2012.

[14] S. B. Maind et al., "Research Paper on Basic of Artificial Neural Network" IJRITCC, 2014.

[15] Vidushi Sharma et al., "A Comprehensive Study of Artificial Neural Networks", IJARCSSE, 2012.

[16] Simon Haykin, "Neural Networks-A Comprehensive Foundation", 2nd Edition, 1999.

[17] M. F. Moller, "A Scaled Conjugate Gradient Algorithm for Fast Supervised Learning", Neural Networks, vol. 6, 1993, pp. 525-533, 1991.

[18] J. Goerlich et al., "Signal Analysis Using Spectral Correlation Measurement", IEEE Instrumentation and Measurement Technology Conference, pp. 1313-1318, 1998

[19] Hong Wang, "Spectral Correlation Function of Basic Analog and Digital Modulated Signals", IEEE, School of Electronic Engineering, University of Electronic Science and Technology of China, 2013.

[20] W.A. Gardner, "Spectral Correlation of Modulated Signals: PART I - Analog Modulation", IEEE Trans. on Communication, vol. 35, pp. 584-594, 1987.

[21] W.A. Gardner, "Spectral Correlation of Modulated Signals: PART II - Digital Modulation", IEEE Trans. on Communication, vol. 35, pp. 595-601, 1987.

[22] F. Z. El Bahi, H. Ghennioui, and M. Zouak, "Performance Evaluation of Energy Detector Based Spectrum Sensing for Cognitive Radio using NI USRP-2930, International Journal of Electrical and Computer Engineering (IJECE), vol. 7, no. 4, pp. 1934 - 1940, August 2017.

[23] Rita Mahajan, Deepak Bagai, "Improved Learning Scheme for Cognitive Radio using Artificial Neural Networks International Journal of Electrical and Computer Engineering (IJECE), vol. 6, no. 1, pp. 257-267. http://www.iaescore.com/journals/index.php/IJECE/article/view/103/119, , February 2016.

[24] H. M. Hamee, J. Wadi, "Automatic Modulation Recognition for MFSK Using Modified Covariance Method" , International Journal of Electrical and Computer Engineering (IJECE), vol 5, no. 3, pp 429-435. http://www.iaescore.com/journals/index.php/IJECE/article/view/5636/4656, , June 2015.

[25] J. Avila, K. Thenmozhi, "Multiband OFDM for Cognitive Radio - A Way for Cyclostationary Detection and Interference Cancellation" International Journal of Electrical and Computer Engineering (IJECE), vol. 6, no. 4, pp. 1702-1709, , August 2016.

\section{BIOGRAPHIES OF AUTHORS}

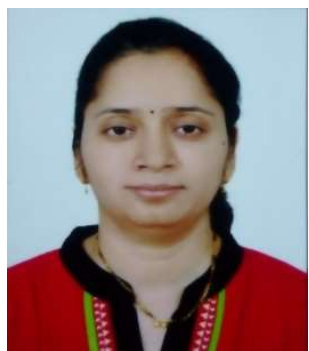

Sheetal D. Borde has completed her bachelor's degree in Electronics and Telecommunications from Government College of Engineering, Aurangabad, Maharashtra, India in year 2001. She completed her Masters degree in Electronics Engineering from Marathwada University, Maharashtra, India in year 2003. Currenlty she is pursuing her Ph. D. in Electronics and Telecommunications engineering from College of Engineering, Pune under Pune University. Having an experience of 14 years, she is currently working as a Assistant Professor in Electronics and Telecommunication Department of PES's Modern college of Engineering, Pune Maharashtra, India. Her research interests include wireless communication, mobile communication and cognitive radio.

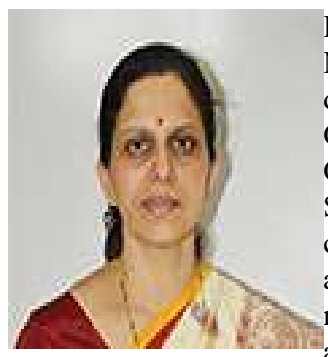

Dr. Mrs. Kalyani R. Joshi has currently been working as Principal and Professor E\&TC in PES's Modern College of Engineering, Pune, Maharashtra, India. She has completed her Bachelor's degree in Electronics Engineering from Shivaji University and Masters degree in E\&TC from College of Engineering, Pune under Pune University. She has got her Ph. D. degree from College of Engineering, Pune in year 2008 with specialization in biomedical image processing. She has been worked as Chair, Signal Processing Chapter, IEEE Pune section for three consecutive years from 2013 to 2015 and awarded as senioer member of IEEE. She has Coauthored a book on Image And Video Compression Fundamentals, CRC Press-2015. She has more than 30 national and international publications in reputed journals and conferences. Her areas of interests are Digital Signal and Image Processing and signal processing in Communications. 